\title{
Automatic anthropometric personalization of a digital human model from a set of subject's photographs
}

\author{
Jean-Pierre Verriest \\ Biomechanics and Impact Mechanics Laboratory, IFSTTAR-Université de Lyon, 25 avenue François Mitterrand, \\ 69500 Bron, France - Email : jean-pierre.verriest@ifsttar.fr
}

\begin{abstract}
The objective of the work is to develop a method for automatic dimensioning of a digital human model (DHM) from a set of calibrated photographs of the subject under study. Fifteen subjects (10 males, 5 females, mean age 27) wearing surface retro-reflective markers at major bony landmarks and standing inside a calibrated space, were photographed by means of low cost main stream digital cameras (face, left and right views). The DHM software used is based on a skeletal structure surrounded by contours defined by cross sections along the skeletal links. Specific points corresponding to bony landmarks are defined in body linkage local reference frames. The photograph calibration method (DLT) allows to superimpose a picture of the DHM on the subject's photographs. A specific algorithm adjusts the length of the manikin skeletal links in function of the measured distance between markers attached to these links (e.g. knee and ankle for the lower leg). Then the contour sections dimensions were adjusted to fit subject's silhouette extracted from the photographs. The results obtained within a few seconds of calculation consist of a personalized DHM representing the subject with an error less than $2 \%$ for stature and less than $4 \%$ for weight.
\end{abstract}

Keywords: Anthropometry, digital human model, personalization, photographic

\section{Introduction}

The biomechanical analysis of a human being in a work situation or interacting with a product can be facilitated by the use of a digital human model (DHM). The relevance of the collected data relies on the accuracy with which the model represents the observed subject from a biomechanical point of view.

Commercially available DHM software offer modules allowing the dimensioning of the manikin from a set of standard anthropometric measures. Besides the time required by the collection of numerous data and the risk of errors introduced by the manual method, the resulting manikin does not always closely match the original subject [1].

The objective of the work presented is to develop an automatic method to derive the dimensions of a numerical manikin (skeleton and contours) from a set of calibrated photographs of the subject under study.

\section{Methods}

\subsection{Subjects}

Fifteen young healthy adults, 11 males and 4 females aged $27 \pm 7$ years, involved in an experiment dealing with movement analysis in a driving task were included in the study. The subjects wore light underwear and 32 retro-reflective markers $(10 \mathrm{~mm}$ diameter) were fixed to the skin surface (or on the clothing in some places) indicating the location of major palpable underlying bony landmarks so that these points could be located in space by means of a motion capture system. The location of the anatomical points was determined according to the guidelines of Van Sint Jan [2].

The main anthropometric dimensions were recorded by standard methods [3] then the subjects were photographed under four views (face, back, left and right) inside a calibrated space bounded by 4 vertical 
calibration bars. These bars bear reflective markers laid according to a different pattern on each bar in order to be automatically identified on the pictures. The subjects were asked to maintain a standing posture with arms and legs slightly off the torso axis.

\subsection{Photographs calibration}

The photographs were taken with low cost mainstream cameras. The resolution was limited to $1500 \mathrm{x}$ 2000 pixels. The calibration is based on the DLT method [4] using the bar markers. For this, the 2D coordinates of these markers on the photographs and the $3 \mathrm{D}$ coordinates in the laboratory frame are needed. The detection of markers on each photograph is automatically obtained by applying a threshold value of 250 for a $0-255$ pixel coding range. Then, the $2 \mathrm{D}$ position of the marker is given by the barycentre of the identified white pixel zone.

\section{3. $3 D$ marker coordinates}

The 3D coordinates of the markers fixed on the subject and on bars were obtained by means of a Vi$\operatorname{con}^{\mathbb{B}}$ motion capture system. But these coordinates can be extracted from the photographs by means of the DLT method if a motion capture is not available. This alternative was used to locate extra body landmarks which were not indicated by surface markers (tragions, nasion and finger tips). The bar marker coordinates can also be known just from a known positioning of the bars.

\subsection{Manikin (DHM)}

Structure : The manikin used (see Fig. 1), Man3D [5], comprises a tree like skeleton whose root is located at the pelvis level and which is composed of 55 links, 52 joints and 102 degrees of freedom (dof), half of these being located in the hands. The contours attached to the links are constituted by triangular facets defined by transverse cross sections along the skeletal link longitudinal axes.

Anatomical points: the manikin comprises also specific points corresponding to the surface markers located indicating the anatomical landmarks. All these points are defined by their coordinates in the local reference frame attached to the skeletal links. These coordinates are normalized with respect to the main dimensions of the body link to which they belong (anterior-posterior depth, lateral width and lon- gitudinal length). A constant term is added in order to introduce the offset of markers with respect to the skin surface. Some of these coordinates have been derived from the literature (Reynolds [6] and Seidel [7] for the pelvis, Salmon [8] for the head) and have a statistical significance.
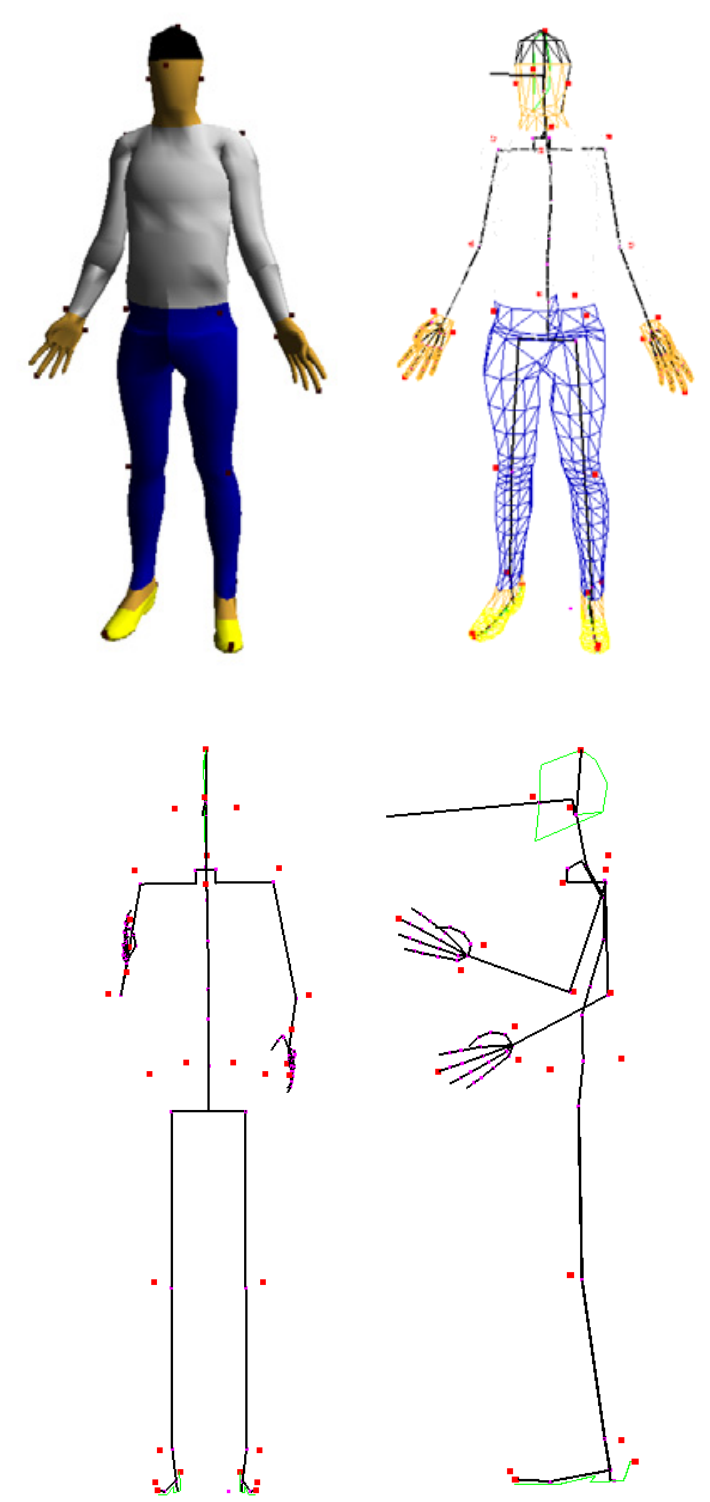

Fig. 1 Man3D, the DHM used to represent the subjects. a) wireframe mode, b) rendered mode, c) stick figure mode with anatomical landmarks

The others have been collected on bone radiographs and don't have any statistical value. The actual coordinates of the markers are obtained by mul- 
tiplying the local normalized coordinates by the dimensioning factor and projecting them in the general reference frame.

The coordinates and dimensioning factors of the anatomical points are given in Table 2 at the end of the document.

\subsection{Body profile}

The subject's body 2D profile was manually created by drawing a polyline matching the body contour on each photograph. It takes 2-3 minutes to click 200-250 points to create the profile line and no specific skill is required. An automatic contour extraction module is being developed in order to avoid this manual operation.

\subsection{Adjustment procedure}

. The calculated DLT parameters allow the calculation of projection parameters of a manikin drawing onto the subject's photograph.

The adjustment consists in modifying first the dimensions, the position and the orientation of the skeletal links of the manikin. In a second phase, the manikin contours are modified in order to fit the subject's profile line on at least two views (face and left). Lateral symmetry of body dimensions is assumed.

Dimensioning principle: In the manikin, all points are defined by their local coordinates normalized with respect to the main dimensions of the body link to which they belong. As shown on Fig. 2, the length $\mathrm{L}$ of a body link (e.g. the shank) can be adjusted as a function of the distance $D_{m}$ between two surface markers $M_{1}$ and $M_{2}$ (e.g. external knee and external ankle markers). These markers correspond to bony landmarks $B_{1}$ and $B_{2}$ respectively. The relationship between $D_{m}$ et $D_{B}\left(D_{B}\right.$ is the distance between $B_{1}$ and $B_{2}$ ) is not necessarily linear and the adjustment is repeated as long as the difference between $D_{m}$ et $D_{B}$ remains greater than a prescribed threshold $\varepsilon$. The corresponding algorithm written in pseudo code is shown in the frame below Fig. 2.

Positioning principle: with at least 3 markers, one can estimate the 3D pose of a body link (see for instance [9] ). But, in the manikin, links are connected to their parent by joints with either 1, 2 or 3 dofs. Knowing the pose of the parent link, according to the number of dofs, one or two markers are enough to determine the pose of the child link.

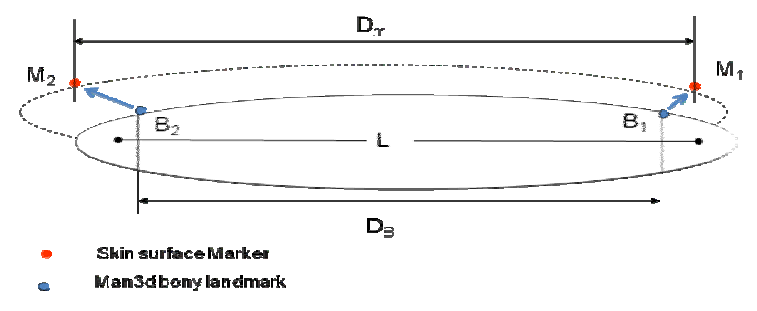

$\mathrm{D}_{\mathrm{m}}=\left\|\mathrm{M}_{1} \mathrm{M}_{2}\right\|$

Do While $\operatorname{abs}\left(\mathrm{D}_{\mathrm{m}}-\mathrm{D}_{\mathrm{B}}\right)>\varepsilon$

$\mathrm{D}_{\mathrm{B}}=\left\|\mathrm{B}_{1} \mathrm{~B}_{2}\right\|$

$\mathrm{K}=\mathrm{D}_{\mathrm{m}} / \mathrm{D}_{\mathrm{B}}$

$\mathrm{L}=\mathrm{L} \times \mathrm{K}$

End do Update $\mathrm{B}_{1}, \mathrm{~B}_{2}$

Fig. 2 Body link dimensioning. $\mathrm{L}$ is the link length, $\mathrm{D}_{\mathrm{m}}$ the distance between two surface markers $\mathrm{M}_{1}$ and $\mathrm{M}_{2}, \mathrm{D}_{\mathrm{b}}$ the distance between the two corresponding bony landmarks $\mathrm{B}_{1}$ and $\mathrm{B}_{2}$. The algorithm used to adjust $\mathrm{L}$ is listed in pseudo code in the lower box.

Sequencing of operations: The dimensioning and positioning process is applied to the body links according to the flow chart of Fig. 3. The process starts at head level then continues by locating the pelvis. At least three points are used for these links. Then, the process goes on with the limbs. On the graph, each step corresponds to a box and the markers used are indicated on the side as input to the box.

Contour adjustment: The components of the body link are projected on the image plane on which the profile line was drawn. For each contour section, a deformation vector $V_{\text {def }}$ is defined in the image plane, perpendicularly to the projection of the longitudinal axis of the body link (see Fig. 4).

The contour points of the section are projected on this vector. The extreme points E1 and E2 on each side of the section allow the calculation of the initial dimension of the projected contour section. The intersections $\mathrm{E}_{1}$ ' and $\mathrm{E}_{2}$, between $\mathrm{V}_{\mathrm{def}}$ and the profile lines define the target dimension for the contour section. Then each point $\mathrm{P}$ of the section is modified according to the change in the dimension. The $\mathrm{V}$ component of vector $\mathrm{CP}(\mathrm{C}$ is the center point of the contour section) along $\mathrm{V}_{\text {def }}$ is multiplied by a coefficient $\mathrm{K}$ equal to the ratio $\left\|\mathrm{CE}^{\prime}\right\| /\|\mathrm{CE}\|$. In fact, contour sections are represented by polygons with 12 vertices for the torso and 8 vertices at limb level. If the photographs are taken along orthogonal axes practically aligned with the body axes, for most of the sections the outmost vertices fall on Vdef.. 


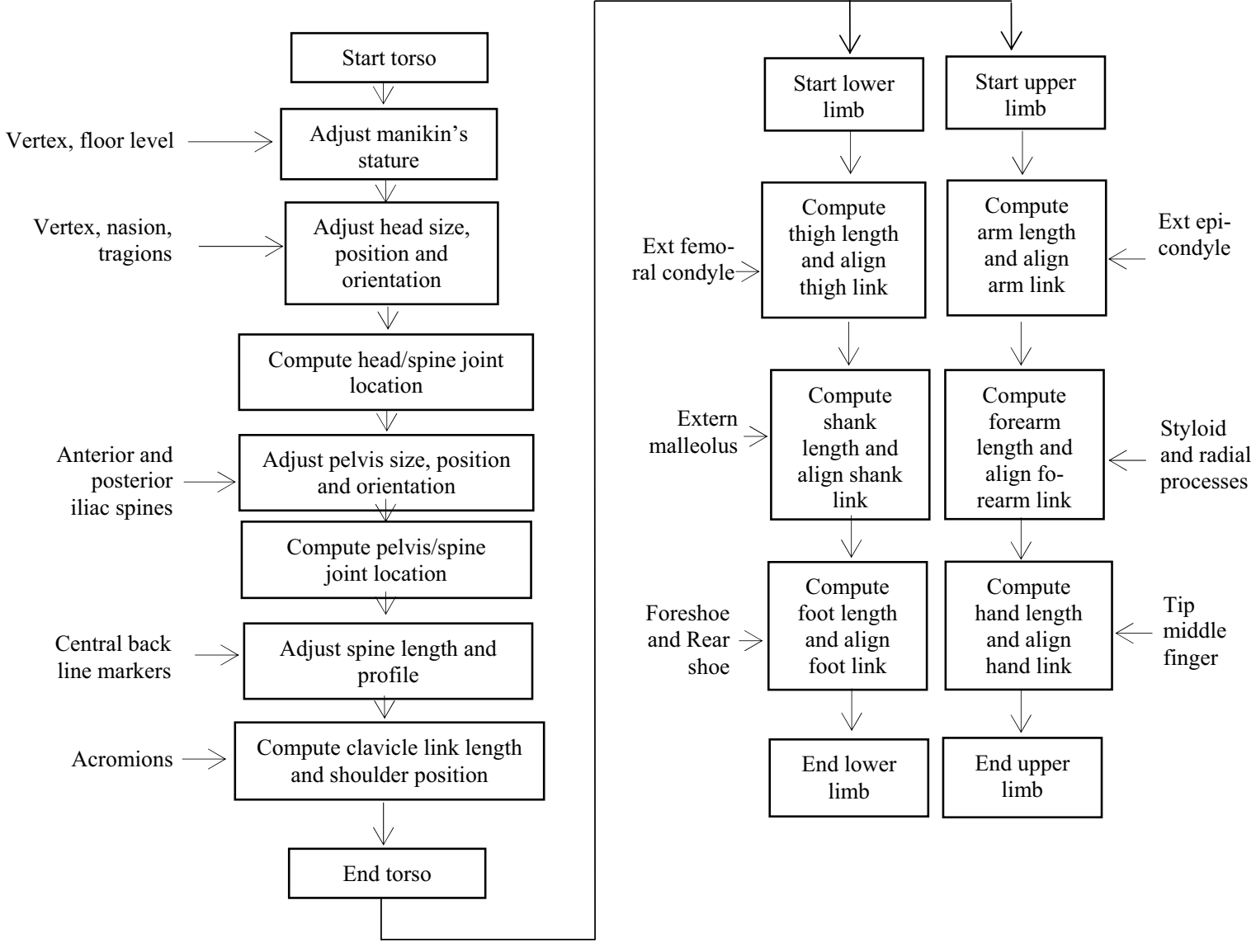

Fig. 3 Flow chart of body link dimensions and position calculation. The input points are indicated on the side of each calculation box.

But if there is some rotation of the link along its longitudinal axis, the outmost vertices are out of $\mathrm{V}_{\text {def }}$ and a small error in the dimensioning occurs The contour adjustment is performed on at least two views, face and left. For the face view, only one profile line is provided. For the side view, two separate lines are provided, one for the torso and lower limb and another one for the upper limb. This will be an issue to solve for the contour extraction module.

Computation of anthropometric dimensions: the anthropometric dimensions are computed between vertices of the adjusted contour mesh and /or anatomical points. The circumferences are estimated by the perimeter of the closest contour section. Two specific postures have been defined, as close as possible to the standard postures for anthropometry measurement. The first is standing with parallel legs and elbows flexed at 90 degrees, the second one is sitting erect with also elbows flexed. Body mass is com- puted from the volume limited by the contours and from a set of volumic mass values found in the literature [10]. These values are listed in Table 1.

\section{Table 1}

Distribution per body region of volumic mass values used to compute body mass

\begin{tabular}{|l|c|}
\hline \multicolumn{1}{|c|}{ Body segment } & Volumic mass $(\mathrm{g} / \mathrm{ml})$ \\
\hline Head & 1.0710 \\
\hline Neck & 1.0420 \\
\hline Thorax & 0.9200 \\
\hline Shoulder & 1.0700 \\
\hline Abdomen/pelvis & 1.0100 \\
\hline Thigh & 1.0500 \\
\hline Shank & 1.0900 \\
\hline Foot & 1.1000 \\
\hline Upper arm & 1.0700 \\
\hline Forearm & 1.1300 \\
\hline Hand & 1.1600 \\
\hline
\end{tabular}




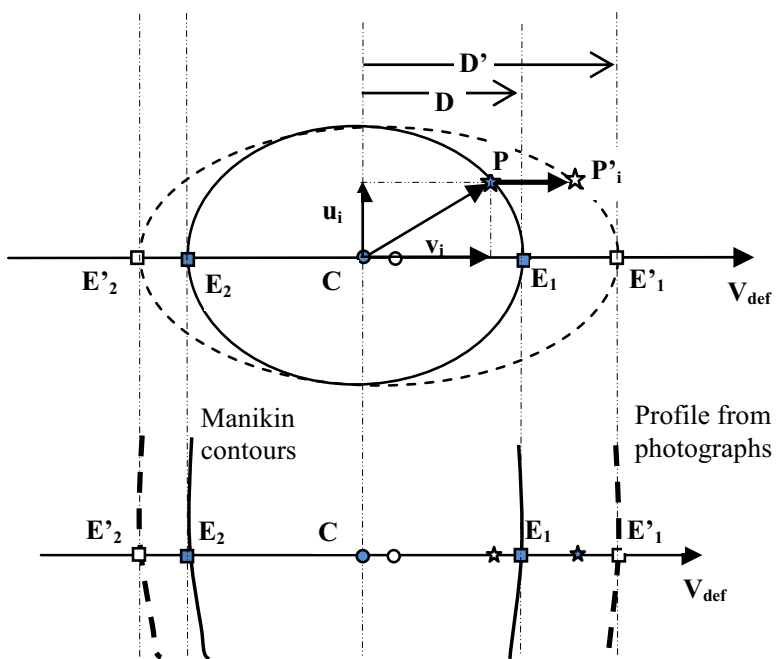

Fig. 4 Adjustment of contour sections against profile extracted from photographs (see text)

\section{Results}

Fig. 5 shows a sample of the results obtained for a male subject. The pictures show from left to right : a) the body silhouette alone; b) the subject's photograph with the silhouette superimposed; c) the skeletal structure adjusted to the subject; d) the adjusted contours in wireframe mode; e) the contours in rendered mode. On average, the position of surface markers is reached with an error of $13.74 \mathrm{~mm}$ (see Fig. 6). The differences between calculated and measured anthropometric values are indicated on the graph of Fig. 7. Stature with shoes on is estimated with a mean deviation of $1.59 \mathrm{~mm}$ but with a standard deviation of 17.7 $\mathrm{mm}$. The maximum error is as high as $33 \mathrm{~mm}$. Body mass is systematically overestimated with an average difference of $2.36 \mathrm{~kg}$ (std. dev.=1.15). The largest deviation is recorded for chest depth which is $28 \mathrm{~mm}$.
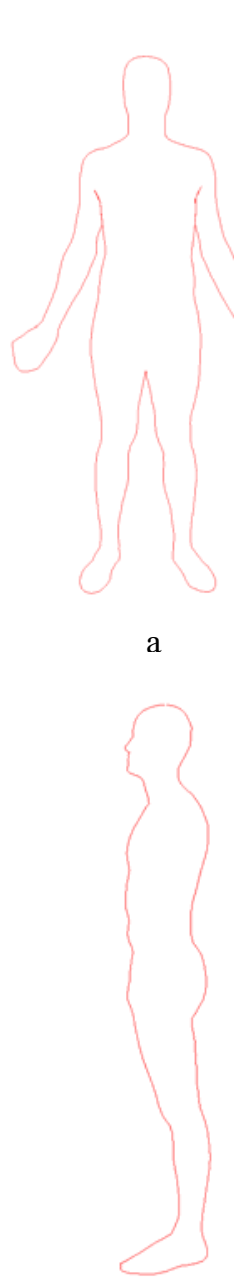

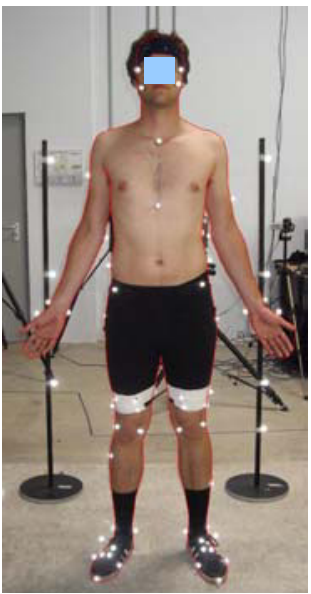

$\mathrm{b}$

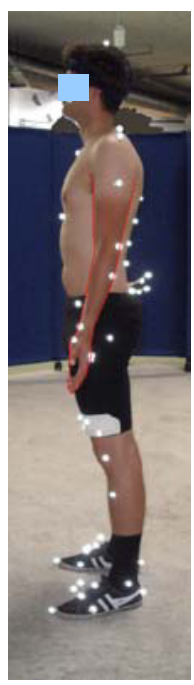

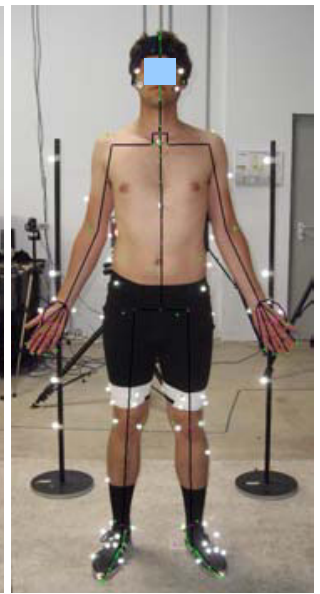

C

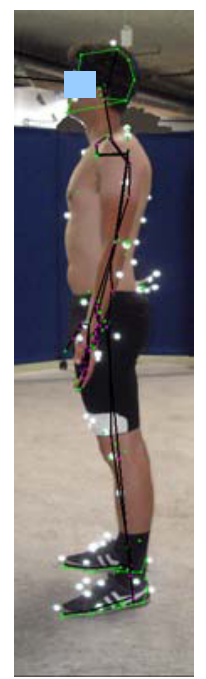

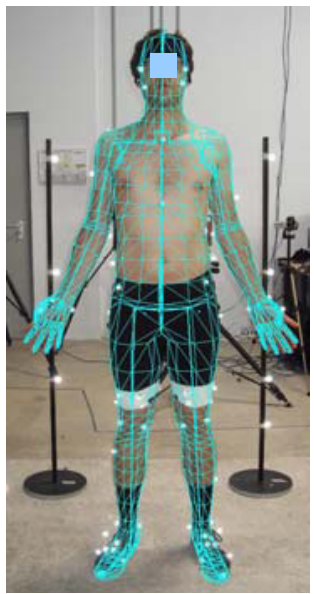

d

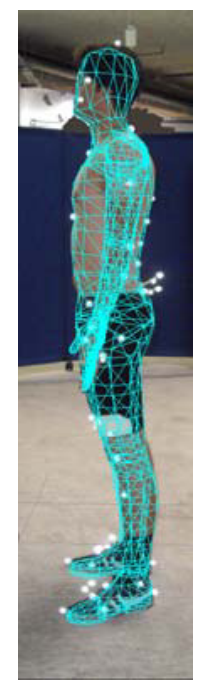

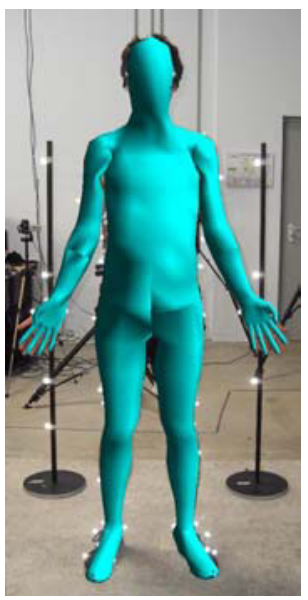

e

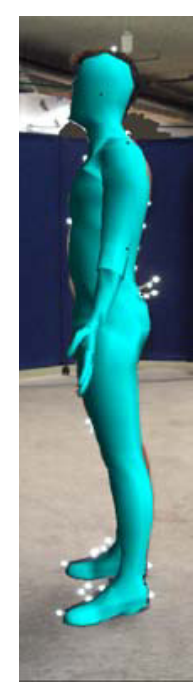

Fig. 5 Illustration of the steps followed in the adjustment process of the posture, dimensions and contours of the manikin. From left to right are displayed: a) the body silhouette alone; b) the subject's photograph with the silhouette superimposed; c) the skeletal structure adjusted to the subject; d) the adjusted contours in wireframe mode; e) the contours in rendered mode 


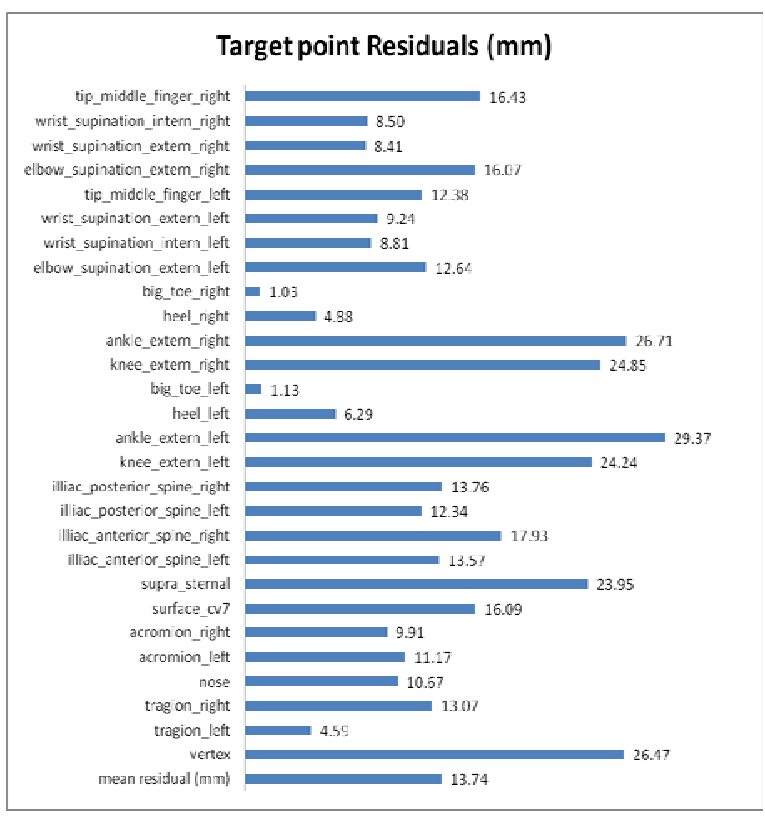

Fig. 7 Position error on calculated target points

\section{Discussion}

Despite the good fit of the adjusted model to the subject's photographs, the computed deviations from target points and anthropometric variables remain important. This is quite surprising because direct measurements of points on the photographs are pretty accurate on the calibration object for instance or on subject's shoes. Several reasons can be mentioned to explain these differences.

Concerning the anatomical points, the assumption was made that they can be calculated by applying to generic normalized coordinates a dimensioning factor along each body segment reference axis. Beside the approximate character of the used values, the natural scatter of that kind of data among the population and the uncertainty about their dependency on the overall dimensions of the concerned body segment probably do not allow reaching the target points with a good accuracy. This assumption is likely insufficient but few data can be found in the literature to support an improvement of this definition. An attempt to derive better values for the 96 dimensioning parameters (32 points $\mathrm{x} 3$ coordinates) from an optimization procedure is being conducted but did not yield results so far.

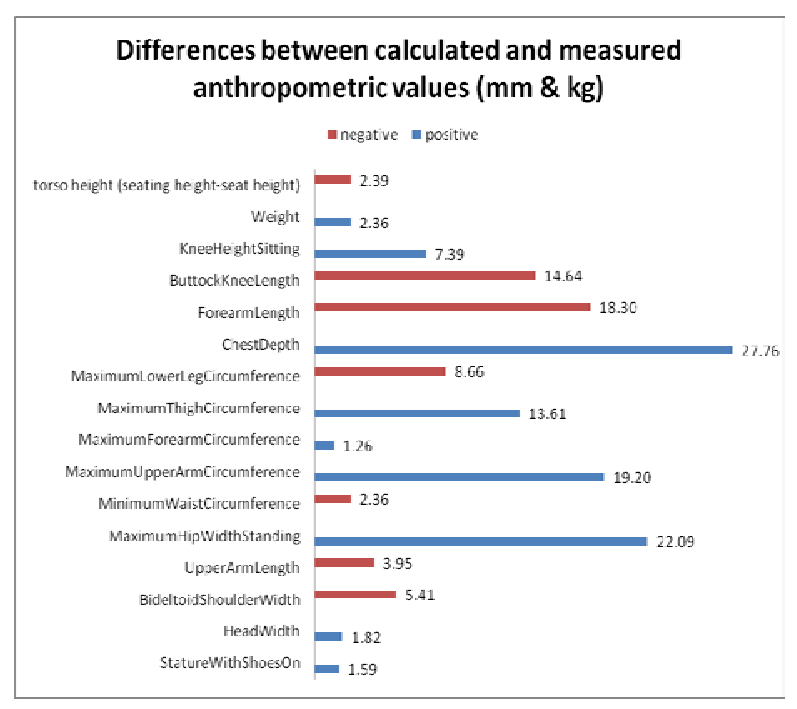

Fig. 6 Error on calculated anthropometric data

The biofidelity of the skeletal model is also a source of errors. Indeed, simplifying hypotheses concerning the joints between certain body segments introduce constraints that can be incompatible. For instance, in the manikin, the elbow flexion axis is assumed perpendicular to the plane passing through the gleno-humeral, the elbow and the wrist joint centers. This provision which neglects the abduction of the forearm relative to the upper arm is not an issue when the purpose is to simulate a generic human. But, when an accurate representation of a real subject is intended, this simplification prevents from meeting the exact geometry. Introducing an inclination of the elbow flexion axis produced a better posture of the upper limb. But this angle, which varies from one individual to another should be part of the adjustment parameter set for personalization.

The anthropometric dimensions calculated on the adjusted mesh are also quite different from those directly measured on the subject's body by standard methods. Several causes can be invoked to explain these differences. The first one is that the mesh of the manikin contours is sparse; as a whole, 1696 vertices define the contour mesh including 576 for both hands only. This low density makes that for several subjects, the minimum waist circumference falls between two torso contour sections. A finer mesh would prevent this type of problem. 
The shape of contour sections is generic and comes from cadaveric data. The adjustment concerns only the main dimensions of the sections but not the position of the vertices. Since, the vertices position condition the body volume, it affects the body mass calculated.

More important, the contour adjustment was made for a specific posture, different from those usually adopted to perform anthropometric measurements. Stature can change if the posture on photographs is more relaxed as compared to the standard measure, back resting on a plane. If other skeletal dimensions are not modified, in principle by posture change, soft tissues dimensions may be changed, especially in the abdomen and pelvis area. Currently, some of the geometric changes of the posterior face of the pelvis area when hip flexion occurs are modeled in the manikin; but this modeling is still insufficient.

The large difference in chest depth measurement found its explanation in the fact that for the standard measurement, subjects were instructed to expire as fully as possible while they were naturally breathing when they were photographed.

The accuracy on calculated anthropometric dimensions is much lower than that obtained with modern full body scanners [11]. But here, the goal is not only to obtain anthropometric dimensions but provide a fully functional sized manikin; this introduces some constraints that are sources of errors.

\section{Conclusion}

A method was developed to automatically adjust the skeleton and contours of a digital human model from at least two photographs of a real subject taken in a calibrated space. The method was applied to a sample of 16 subjects, male and females and provided a realistic representation of these subjects. The accuracy on the anatomical point position and on the calculated anthropometric dimensions can probably be improved through a better definition of the coor- dinates of anatomical landmarks and through the use of a finer and more realistic mesh.

\section{References}

[1] Alexander, T., Conradi, J., Analysis of anthropometry and range validity of the digital human model RAMSIS, 2001, SAE paper 2001-01-2104, Society of Automotive Engineers, inc. Warrendale, Pa., USA

[2] Van Sint Jan, S., Color atlas of skeletal landmark definition : guidelines for reproducible manual and virtual palpation. Churchill Livingstone ed., 2007

[3] ISO 7250: Basic human body measurements for technological design, International Organization for Standardization, 1998.

[4] Abdel-Aziz, Y.I., Karara, H.M., Direct Linear Transformation from comparator coordinates into object space coordinates in close-range photogrammetry, in : Proceedings of the ASP/UI Symposium on close-Range Photogrammetry,Urbana, Illinois, USA, January 1971

[5] Verriest J.P., MAN 3D - A functional and geometrical model of the human operator for computer aided ergonomic design, in : Advances in Industrial Ergonomics and Safety, W. Karwowski \& J.W. Yates ed., Proc. IFIESR Congress, Lake Tahoe, USA, Taylor \& Francis, 1991, pp. 901-908.

[6] Reynolds, H.M., Snow, C.C., Young, J.W., Spatial geometry of the human pelvis. Report AAC-119-81-5, Protection and survival laboratory, Civil aeromedical Institute, FAA, Oklahoma City, USA

[7] Seidel, G.K., Marchinda, D.M, Dijkers, M., Soutas-Little, R.W., Hip joint center location from palpable bony landmarks - A casaver study, J. Biomech., 28, 8, pp 995-998, 1995

[8] Salmon, J.P., Différences sexuelles d'éléments cranio-faciaux osseux et cutanés chez l'enfant et l'adulte jeune en orientation vestibulaire. Thèse de doctorat en chirurgie dentaire, Université de Lille, 1972.

[9] Spoor, C.W., Veldpaus, F.E., Rigid body motion calculated from spatial co-ordinates of markers. 1980, J Biomech 13, 391-393.

[10] Clauser C.E, McConville J.T, Young J.W. (1969) Weight, Volume and Center of Mass of Segments of the Human Body. AMRL Technical Report 69-70, Wright patterson Air Force Base, Ohio, USA

[11] Kouchi, M., Mochimaru, M., Evaluation of accuracy in traditional and 3D anthropometry, 2008, SAE technical paper 2008-01-1882, Society of Automotive Engineers, inc. Warrendale, $\mathrm{Pa}$., USA

Acknowledgements : Many thanks to Jeanne Bulle, PhD student, for providing photographs and anthropometric data. Thanks also to Ingrid Bubb, intern student, for her help in defining the anatomical point location. 
Table 2

Definition of the anatomical landmarks and user points coordinates in the local skeletal link reference frames. Local coordinates are obtained by multiplying the coefficient by the dimensional parameter value. $(\mathrm{D}=$ anterior-posterior depth, $\mathrm{W}=$ lateral width, $\mathrm{Z}=$ length of the skeletal link

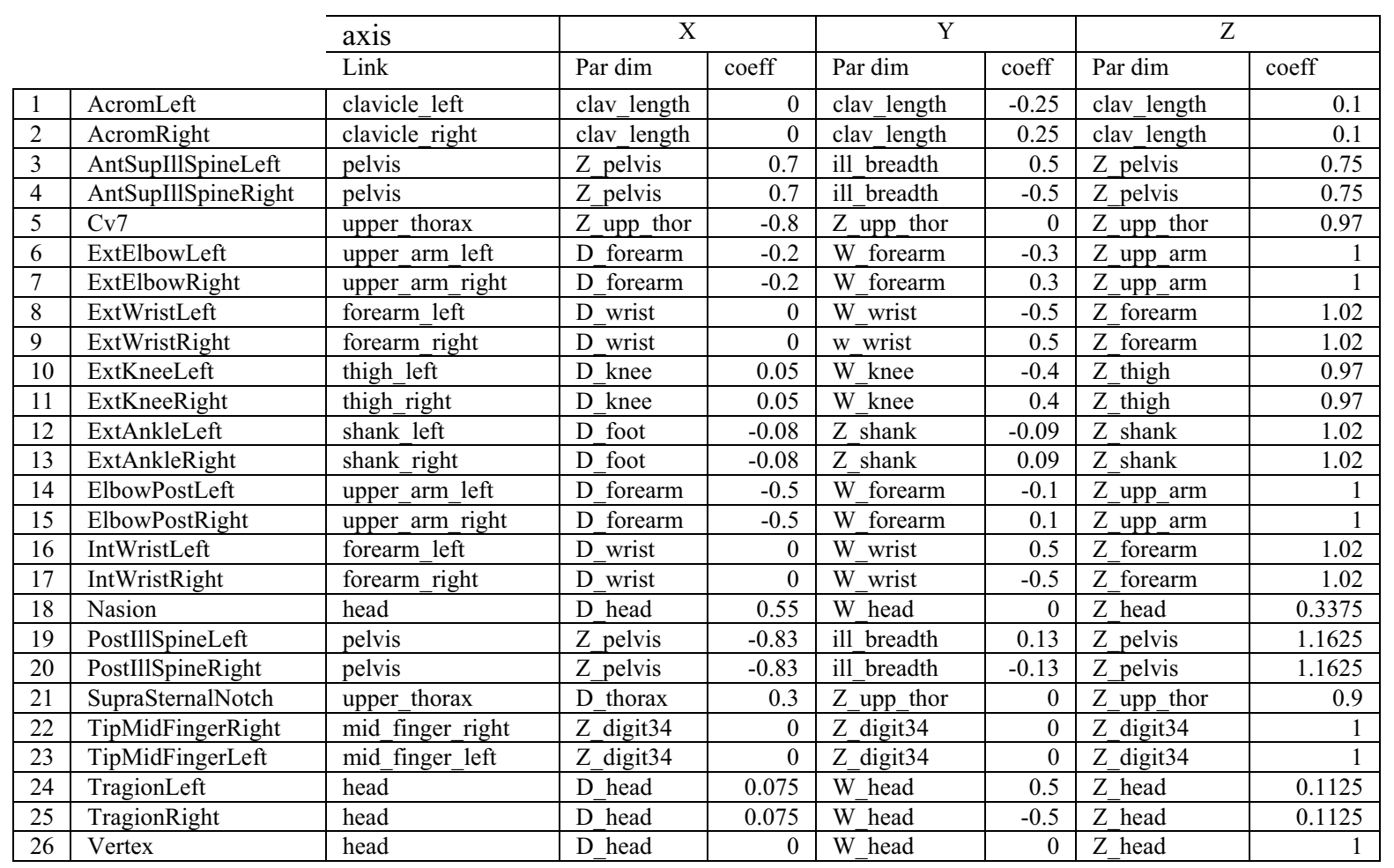

